\title{
Medium-scale traveling ionospheric disturbances over Taiwan observed with HF Doppler sounding
}

\author{
Jiří Fišer ${ }^{1} 2^{*}\left(\mathbb{0}\right.$, Jaroslav Chum ${ }^{1}$ (1) and Jann-Yenq Liu ${ }^{3}$
}

\begin{abstract}
We analyzed horizontal velocities of medium-scale traveling ionospheric disturbances using HF Doppler sounding over Taiwan from January 2014 to January 2016. Meridional components of the observed horizontal velocities are in most cases larger than zonal components. In summer, poleward propagation prevails, whereas in winter, disturbances primarily propagate southward. Zonal components of the analyzed events are mostly eastward. Seasonal behaviors of the observed propagation directions are similar at other locations with HF Doppler sounding at middle and low latitudes. Horizontal velocities of the observed events are in the $50-400-\mathrm{ms}^{-1}$ range. The upper limit is determined using the spacing between reflection points, making the uncertainty in velocity very large for events with velocities larger than this limit. The mean horizontal velocity is $210 \mathrm{~ms}^{-1}$. The range between the 20th and 80th percentile of horizontal velocities is $144-281 \mathrm{~ms}^{-1}$.
\end{abstract}

Keywords: lonosphere, MSTIDs, HF Doppler sounding

\section{Introduction}

Traveling ionospheric disturbances (TIDs) are wavelike perturbations of electron density that propagate in the ionosphere. Hines (1960) showed that they are usually caused by internal gravity waves (GW/s) that propagate in the thermosphere. According to Hunsucker (1982), TIDs can be classified as small, medium, and large scale. MSTIDs are waves with velocities of $250-1000 \mathrm{~ms}^{-1}$, periods $15 \mathrm{~min}$ to $\sim 1 \mathrm{~h}$, and wavelengths of several $100 \mathrm{~km}$.

GWs that cause MSTIDs have various proposed origins. GWs have been reported as caused by meteorological processes in the troposphere, such as the jet stream (Bertin et al. 1978; Waldock and Jones 1987; Wautelet and Warnant 2015), vortexes (Wan et al. 1998), typhoons (Huang et al. 1985), or convective systems (Lay et al. 2015; MacDougall et al. 2011). The propagation of GWs from the troposphere to the thermosphere may be

\footnotetext{
*Correspondence: jifi@ufa.cas.cz

${ }^{1}$ Institute of Atmospheric Physics, Czech Academy of Sciences, Bocni II 1401, Prague, Czech Republic

Full list of author information is available at the end of the article
}

indirect. As the air density decreases with height, due to energy conservation laws, the wave amplitudes increase so that quasi- and nonlinear effects appear and wave breaking may occur. This breaking imposes body forces on the thermosphere and produces secondary GWs with larger vertical scales and higher phase speeds (Fritts et al. 2011). GW propagation from below is also influenced by thermal ducts that can transfer wave-associated energy and momentum over large horizontal distances (Hocke and Schlegel 1996; Yu and Hickey 2007). Another mechanism for generating GWs is the solar terminator, when gradients in the thermosphere arise and wave activity is a mechanism for reversing to the equilibrium state (Boška et al. 2003; Šauli et al. 2006). Auroral processes also generate GWs, which propagate equatorward (Hunsucker 1982). Other proposed processes for generating GWs include tsunamis (Artru et al. 2005) and solar eclipses (Altadill et al. 2001). When GWs propagate in the thermosphere, they interact with ionospheric free electrons via collisions; therefore, it is possible to observe them using ionosphere remote sensing methods. MSTIDs associated with gravity waves might be damped 
at midlatitudes at nighttime, except those that propagate in the Perkins direction (Kelley 2011).

MSTIDs may affect radio astronomical measurements and the accuracy of global navigation satellite systems (GNSSs) (Hernández-Pajares et al. 2012). Hence, including their parametrization into atmospheric models is required (Liu et al. 2014). They also play an important role in the energy budget of the lower thermosphere, and transport momentum and energy from lower to higher atmospheric layers (Laštovička 2009).

Recently, MSTIDs have been studied statistically in many places worldwide using various techniques (Frissell et al. 2014, 2016; Fukushima et al. 2012; Grocott et al. 2013; He et al. 2004; Ichihara et al. 2013; Ishida et al. 2008; Klausner et al. 2009; Kotake and Otsuka 2007; Medvedev et al. 2013; Oinats et al. 2015, 2016; Otsuka et al. 2011, 2013; Shiokawa et al. 2003, 2009). In this study, we present observations of MSTIDs' horizontal velocities over Taiwan using HF Doppler sounding. The HF Doppler radar can sense altitudes in the 146-350-km range, depending on time of day, season, and solar activity. The role of neutral winds is also discussed.

\section{Experimental}

\section{HF Doppler sounding}

HF Doppler sounding is a method for remote sensing of the ionosphere. Radio waves transmitted from the ground with very stable frequency are reflected by the ionosphere at the point where the frequency of the wave equals the local plasma frequency, and then are received by the ground receiver. The frequency of the received wave is Doppler shifted. The Doppler shift $\Delta f$ is determined from the time derivative of the sounding radio wave's phase path integrated along the ray path:

$$
\Delta f=-2 \cdot \frac{f}{c} \frac{\mathrm{d}}{\mathrm{d} t} \int_{0}^{z_{\mathrm{R}}} \mu \cdot \mathrm{d} r=-2 \cdot \frac{f}{c} \int_{0}^{z_{R}} \frac{\partial \mu}{\partial N} \cdot \frac{\partial N}{\partial t} \cdot \mathrm{d} r,
$$

where $N$ is electron density, $f$ is the sounding signal frequency, $\mu$ is the real part of its refractive index, $c$ is the speed of light in a vacuum, and $z_{\mathrm{R}}$ is the height of reflection.

Because the refractive index depends on the magnetic field and electron density, perturbations in both cause perturbations in the observed Doppler shift. Provided that the electron density changes due to photoionization and electron loss processes can be neglected, there are three main mechanisms causing the Doppler shift (Sutcliffe and Poole 1989): (1) "magnetic mechanism," caused by the oscillations of the magnetic field, (2) "advection" mechanism, associated with the reflection level's vertical motion, and (3) "compression" mechanism, due to the rarefaction and compression of electron gas. Both the magnetic and compression mechanisms can be neglected for GWs. The Doppler shift caused by the advection mechanism can be interpreted as caused by the movement of the reflection level with velocity $v$, which is calculated using the simple formula:

$$
\Delta f=-2 f \frac{v}{c} .
$$

GWs propagating in the ionosphere cause an undulating electron density profile, which causes a variation in the reflection height, and thus a variation in the Doppler shift. If the radius of curvature of the perturbation is smaller than its mean height, three echoes with different Doppler shifts are observed at the same time, corresponding to three different points of reflection. In the spectrogram, these events form a characteristic "S" shape (Davies and Baker 1966). Such signals require manual approach or approximations. If the radius of curvature of the perturbation is larger than its mean height, the Doppler shift forms a narrow trace with a wavelike shape in the Doppler shift spectrogram. The signals are singlevalued functions of time and can be analyzed directly. In the case of spread F, the Doppler shift traces become very diffusive with a wide range of Doppler shifts present at the same time, corresponding to multipoint reflections from many small-scale irregularities. In the case of a sporadic E layer, the reflected wave has nearly zero Doppler shift and forms a straight line in the Doppler shift spectrogram. GW properties cannot be determined at the time of spread F or sporadic E layer occurrence.

\section{Doppler sounding system}

Our study is based on measurements using a continuous Doppler sounding system installed in Taiwan. This system consists of three transmitters with a fixed highly precise frequency of $\sim 6.57 \mathrm{MHz}$ and two receivers; Table 1 lists their locations. The frequency of each transmitter differs by a few $\mathrm{Hz}$ from others for convenient visualization in one spectrogram. After reception, the signal is down-converted in frequency. The down-converted

\begin{tabular}{llll}
\multicolumn{4}{l}{$\begin{array}{l}\text { Table } 1 \\
\text { Positions of receivers }\end{array}$} \\
for the HF Doppler sounding system in Taiwan \\
\hline Code & Location & Latitude $\left({ }^{\circ} \mathrm{N}\right)$ & Longitude $\left({ }^{\circ} \mathrm{E}\right)$ \\
\hline $\mathrm{R} \times 0$ & NCU Zhongli & $24^{\circ} 58^{\prime} 19^{\prime \prime}$ & $121^{\circ} 11^{\prime} 30^{\prime \prime}$ \\
$\mathrm{R} \times 1$ & Chi Nan University & $23^{\circ} 57^{\prime} 18^{\prime \prime}$ & $120^{\circ} 55^{\prime} 37^{\prime \prime}$ \\
$\mathrm{T} \times 1$ & $\quad$ National Dong Hwa & $23^{\circ} 53^{\prime} 50^{\prime \prime}$ & $121^{\circ} 33^{\prime} 3^{\prime \prime}$ \\
$\mathrm{T} \times 2$ & $\quad$ University & & \\
$\mathrm{T} \times 3$ & Sanyi & $24^{\circ} 20^{\prime} 26^{\prime \prime}$ & $120^{\circ} 46^{\prime} 41^{\prime \prime}$ \\
\hline
\end{tabular}


signal is sampled with a frequency of $305 \mathrm{~Hz}$. This allows us to obtain a Doppler shift with the time resolution of approximately $10 \mathrm{~s}$ and frequency resolution of several $0.01 \mathrm{~Hz}$. The exact resolution depends on the specific time window that the spectral analysis is performed. With this configuration, three points for each receiver in the ionosphere exist from which the sounding signals reflect. The height of reflection varies throughout the day as the ionospheric conditions change, and can be determined from the ionosonde operating in the system area. When the sounding signal frequency is greater than the ionospheric critical frequency of the extraordinary mode, the reflection does not occur and no signal is observed. In the ideal case of an unperturbed horizontally stratified ionosphere, this point would lie in the middle between a transmitter and receiver.

The locations of transmitters and receivers in Taiwan are shown in Fig. 1. A more detailed description of similar Doppler sounding systems operating in other locations can be found in Chum et al. $(2010,2012)$, and Doppler sounding theory is described in more detail in Chum et al. (2016).

\section{Data analysis}

Horizontal velocities of MSTIDs observed using HF Doppler soundings are calculated from time delays between the Doppler shifts observed on signals from different transmitter receiver pairs. The signal from each transmitter reflects from the ionosphere at a different point. Therefore, the perturbation's horizontal movement causes a signature in the Doppler shift, which is

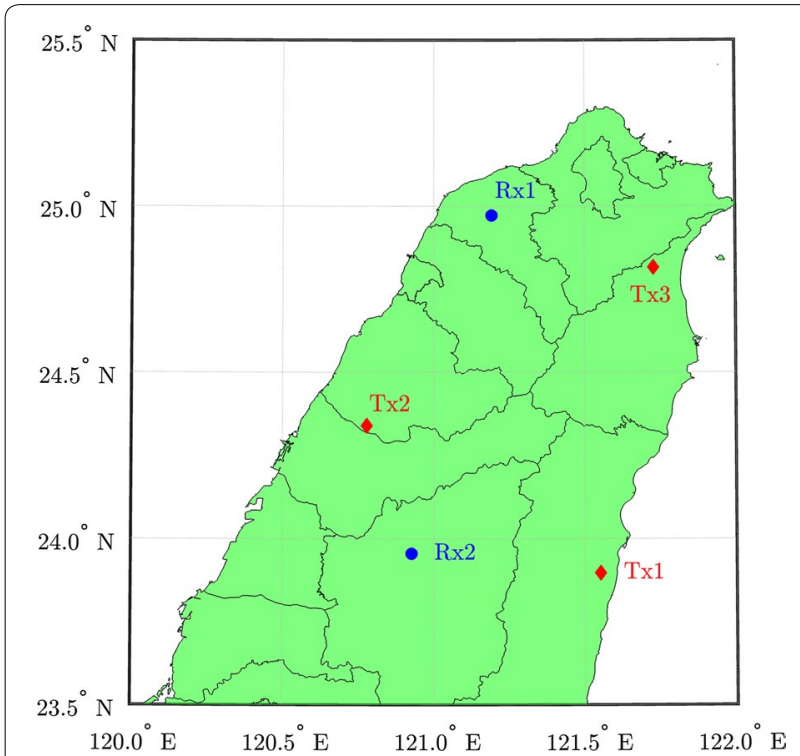

Fig. 1 Locations of the ionospheric Doppler sounding system receivers and transmitters in Taiwan observed on each sounding trace at a different time. With knowledge of time differences between observations on each trace and an estimate of reflection point positions, it is possible to calculate the horizontal velocity of the perturbation.

The process for analyzing the measured data is described as follows. In the first step, the Gabor transform is applied to the signal from the receiver, and a spectrogram is calculated. Traces of three sounding signals are present in the spectrogram. Next, a time series of Doppler shift is obtained for each trace by searching for maximum spectral density. When an $\mathrm{S}$ shape is present in the spectrogram, i.e., multiple frequencies on one trace at the same time, it is approximated using a steplike transition in the time series. For our analysis, we use 90-min-long spectrograms beginning at each hour of the day. Therefore, the intervals overlap by $30 \mathrm{~min}$. It was not possible to obtain a time series of Doppler shift for each interval. The signal is missing if the sounding frequency is larger than the critical frequency of the ionosphere. It may be also missing in the case of an extremely large attenuation in the D layer during high solar activity. In addition, the Doppler shift may become very diffusive in the case of spread F occurrence, so it is impossible to approximate the received Doppler shifts with a singlevalued function of time. An example spectrogram and fitted time series are provided in Fig. 2.

In the second step, we performed a slowness analysis of the fitted Doppler shift time series. This is a method for determining plane wave propagation directions, when measurements are conducted with a field of spatially separated instruments (Johnson et al. 2011). The method is based on shifting the signals measured at each point

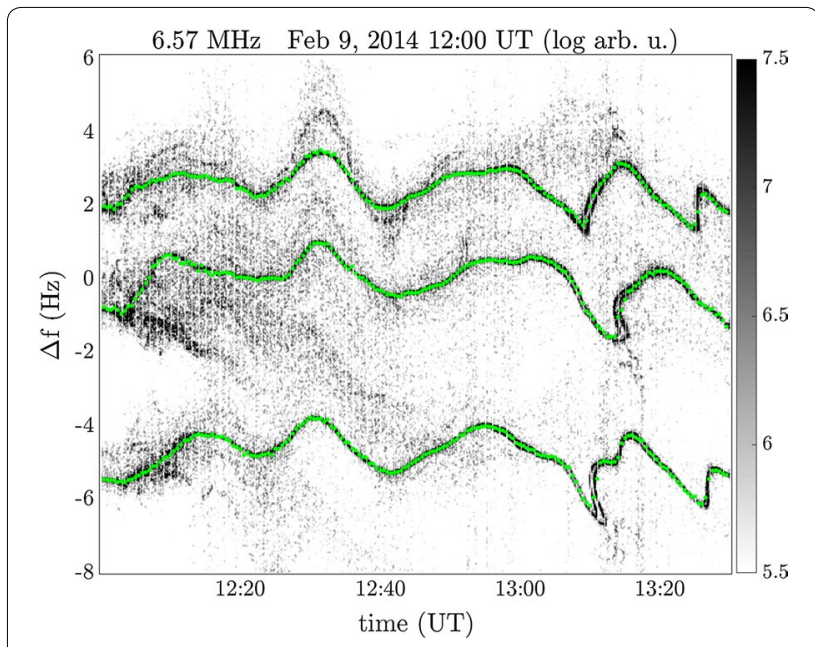

Fig. 2 Example of a Doppler shift spectrogram analyzed for one 90-min interval starting on February 9, 2014, at 12:00 UT. Green dots depict the fitted Doppler shift time series 
in the array to the reference point with various values of slowness, the inverse of velocity, and summing them together. This method has been successfully used for analyzing GW propagation in Chum et al. (2014), who also verified that the obtained propagation velocities were consistent with those observed with other methods. In the 2-D case, the outcome is a two-dimensional function of slowness components $W\left(s_{x}, s_{y}\right)$, which is calculated as:

$$
W\left(s_{x}, s_{y}\right)=\sum_{i=1}^{N_{S}}\left[\sum_{n=1}^{N} \frac{f_{D n}\left(t_{i}+s_{x} \Delta x_{n}+s_{y} \Delta y_{n}\right)}{N}\right]^{2},
$$

where $t_{i}$ is the time of measurement of the $i$ th sample, $s_{x}$ and $s_{y}$ are, respectively, the northward and eastward components of slowness, $f_{D n}$ is the Doppler shift time series from the trace of the $n$th transmitter, and $\Delta x$ and $\Delta y$ are, respectively, the northward and eastward distances of the $n$th reflection point from the center of the array. This center is calculated as the center of mass of the triangle formed by the estimated reflection points of each trace. $N$ is the number of traces (transmitters) and $N_{s}$ is the discrete length of Doppler shift time series. The function $W\left(s_{x} s_{y}\right)$ can be normalized approximately to the range [0, 1] as $C\left(s_{x^{\prime}} s_{y}\right)$ (Chum et al. 2014):

$$
C\left(s_{x}, s_{y}\right)=\frac{W\left(s_{x}, s_{y}\right)}{\frac{1}{N} \sum_{n=1}^{N}\left[\sum_{i=1}^{N_{s}} f_{D n}\left(t_{i}\right)^{2}\right]} .
$$

An example of a normalized function $C\left(s_{x} s_{y}\right)$, calculated for the interval displayed in Fig. 2, is displayed in Fig. 3. The maximum of the function, which in this case corresponds to the velocity of $267 \mathrm{~ms}^{-1}$ and bearing of $140^{\circ}$, is considered the best estimate of the disturbance's horizontal propagation parameters. The parameter uncertainties are determined by calculating the parameters over slightly different time intervals (Chum et al. 2014). This method is applied to all selected intervals. The Doppler shift series are band-pass filtered to the 5-45-min period range. For the final evaluation of results, the following limiting conditions are used. (1) We include only those events where the value of the normalized energy's maximum exceeds 0.6 and root mean square of the Doppler shift signal averaged over all three traces exceeds $0.06 \mathrm{~Hz}$. (2) We exclude intervals that have a slowness uncertainty larger than $2.1 \mathrm{skm}^{-1}$. (3) We exclude intervals where the velocity of the TIDs propagation position is larger than $400 \mathrm{~ms}^{-1}$. For larger velocities, the uncertainties in the parameter estimations are too large because of the relatively small distances between the reflection points, $\sim 50 \mathrm{~km}$, which creates short time delays approaching the time resolution.

HF Doppler soundings have been used to investigate MSTIDs for a long time, e.g., Georges (1968) and Crowley

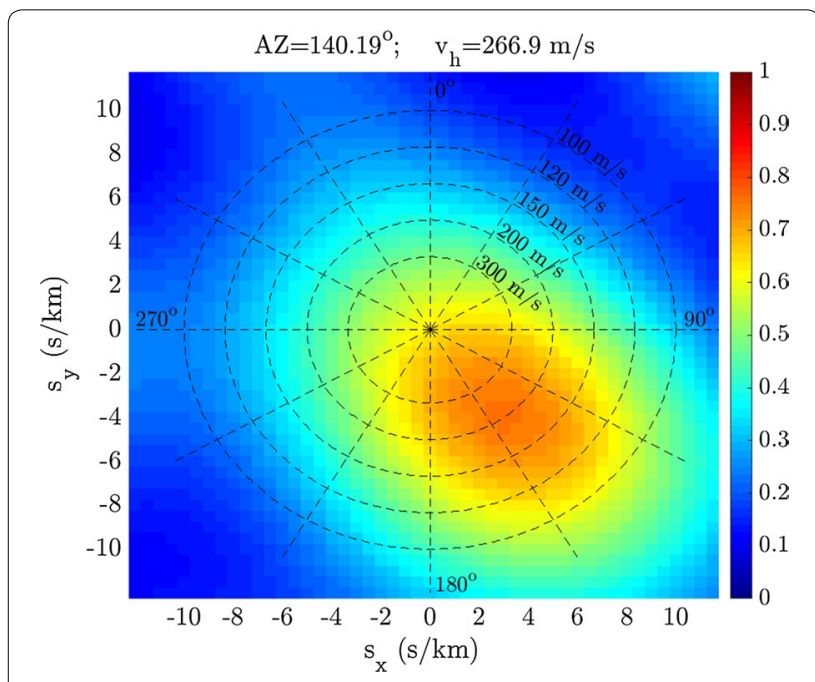

Fig. 3 Normalized energy map calculated using the slowness method from the Doppler shift time series obtained for the 90-min interval starting on February 9, 2014, at 12:00 UT

and Rodrigues (2012). Another method for MSTIDs observations, often used because of GPS receiver network availability, is GPS-TEC measurement. We compared our measurements with GPS-TEC observations during Super Typhoon Meranti on September 13, 2016, presented in Chou et al. (2017). The authors observed several events with concentric TIDs generated from the typhoon propagating northwestward. As an example, an analysis of one 90-min-long interval starting at 9:00 UT on September 13 is presented in Fig. 4. This interval corresponds to the third wave train in Fig. 3 in Chou et al. (2017). The horizontal propagation direction of the waves observed using HF Doppler sounding is $319^{\circ}$, which corresponds with the northwestward propagation observed with the GPS-TEC. The observed horizontal propagation velocity determined using the HF Doppler sounding is about $108 \mathrm{~ms}^{-1}$. Chou et al. (2017) observed velocities of $\sim 157 \mathrm{~ms}^{-1}$ for the wave front at approximately 9:00 UT. This velocity gradually slowed to $\sim 106 \mathrm{~ms}^{-1}$ at around 11:00 UT. The discrepancies between the velocities observed using the HF Doppler and GPS-TEC methods can be ascribed to the GPS-TEC being primarily sensitive to the fluctuations around the peak of the ionosphere, whereas the HF Doppler sounding senses lower heights. Neutral winds that influence the observed MSTIDs might be different at individual observation heights.

\section{Results}

We mainly analyzed measurements from receiver Rx1 from January 2014 to January 2016 as it provided more reliable data than $\mathrm{Rx} 0$. Unfortunately, this period contained many data gaps due to non-operation of at least one 

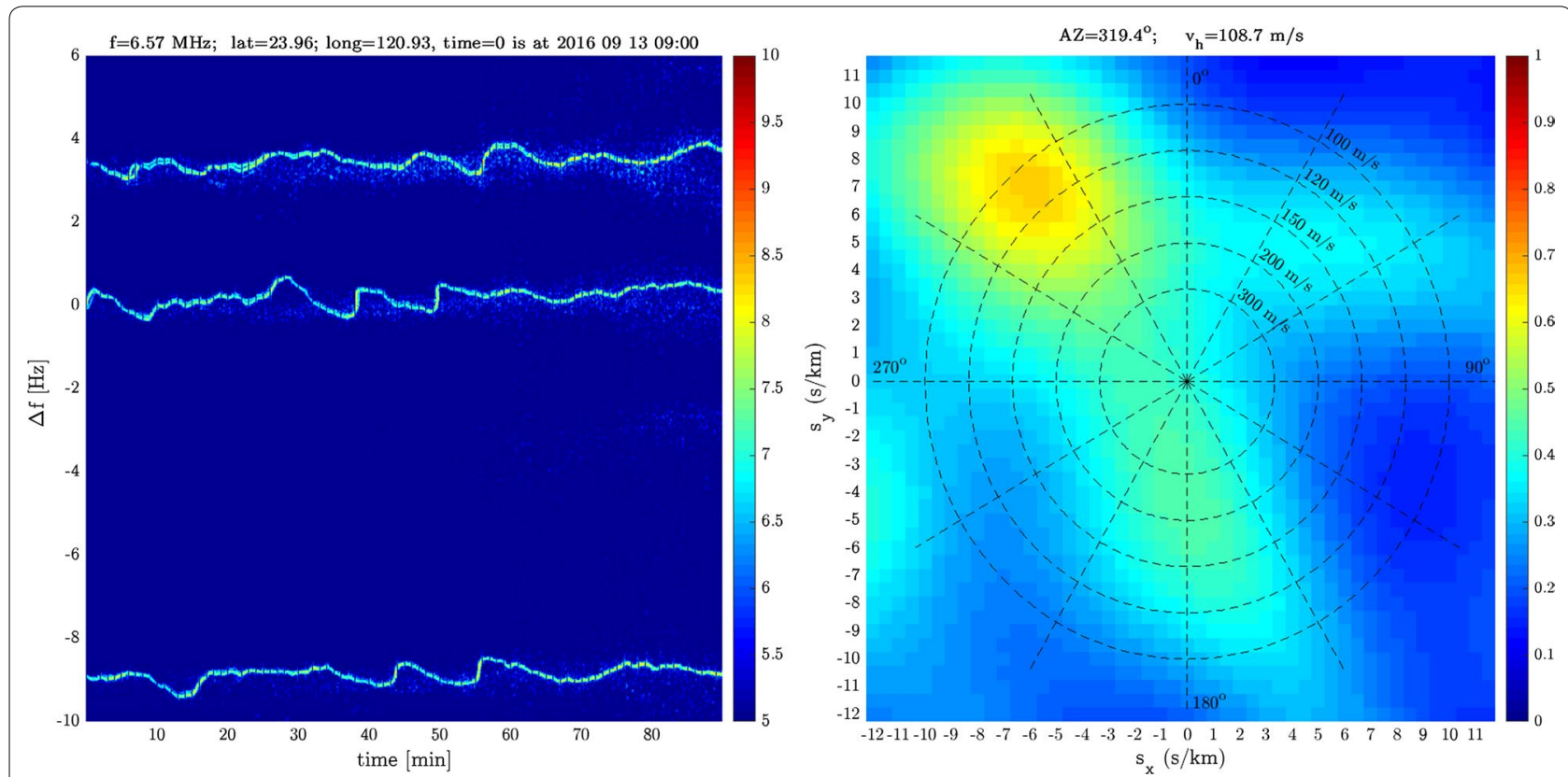

Fig. 4 (Left) an example of a Doppler shift spectrogram analyzed for one 90-min interval analyzed during Super Typhoon Meranti starting on September 13, 2016, at 9:00 UT. (Right) a normalized energy map calculated using the slowness method from the Doppler shift time series obtained for the observations shown on the left

transmitter or receiver or the receiver worked incorrectly. If reliable measurements from Rx1 were missing we used measurements from receiver $\mathrm{Rx} 0$ if they were available. In total, 1258 90-min-long intervals that met the conditions described in the previous section were found. The observation height range calculated using the International Reference Ionosphere (IRI) 2012 model (Bilitza et al. 2014) is $146-350 \mathrm{~km}$. Criteria chosen for selecting events described in "Data analysis" subsection led to a non-uniform distribution in the analyzed GWs with respect to LT and season, which influenced the obtained statistical results.

Figure 5 depicts distributions of the observed MSTIDs' horizontal velocities, maxima in $C\left(s_{x} s_{y}\right)$, and root mean square of the Doppler shift. The velocities of the observed GWs range from 50 to $400 \mathrm{~ms}^{-1}$, which is the upper limit for selection. The mean value of horizontal velocity is $210 \mathrm{~ms}^{-1}$, the 20th percentile is $144 \mathrm{~ms}^{-1}$, and the 80th percentile is $281 \mathrm{~ms}^{-1}$.

A distribution of all observed bearings is depicted in Fig. 6. MSTIDs propagate in all directions, except the sector between $\sim 180^{\circ}$ and $300^{\circ}$. The zonal component of horizontal velocity is eastward for most events, and the meridional component is larger in most cases.

The distribution of MSTIDs bearings in the three different seasons-summer (MJJ-May, June, July, August), winter (NDJ-November, December, January, February), and around equinoxes (March, April, September, October)-is depicted in Fig. 7. In summer, MSTIDs propagate mostly with directions ranging from $0^{\circ}$ to $60^{\circ}$, i.e., north to northeast. In winter, MSTIDs propagate mostly to the southeast with bearings around $150^{\circ}$. In the equinoctial season, the meridional component of the MSTIDs horizontal velocity is northward for most events. The zonal component is preferentially eastward.

Figure 8 depicts bivariate daytime azimuth histograms for different seasons. The observation time for each event corresponds to the middle of an analyzed 90-min interval. The number of events in each bin is indicated with the darkness of gray color. The dashed red line depicts the total number of events observed in each hour normalized to the range $(0,360)$. Most events occur in late afternoon, except in the equinoctial season, when the occurrence of MSTIDs peaks after midnight. The low number of observations past midnight and at early morning might be due to a lower critical frequency of the ionosphere than sounding frequency.

Similar seasonal behavior, i.e., poleward propagation in summer and equatorward in winter, was observed in previous studies using HF Doppler sounding over the Czech Republic, South Africa, and Argentina (Chum et al. 2010, 2012, 2014). Ding et al. (2011) investigated MSTIDs over Central China using GPS-TEC measurements. This region is $\sim 1000 \mathrm{~km}$ from Taiwan. They found that MSTIDs in winter occurred mostly in daytime and the meridional component of their propagation direction was equatorward. In comparison, MSTIDs in summer occurred 

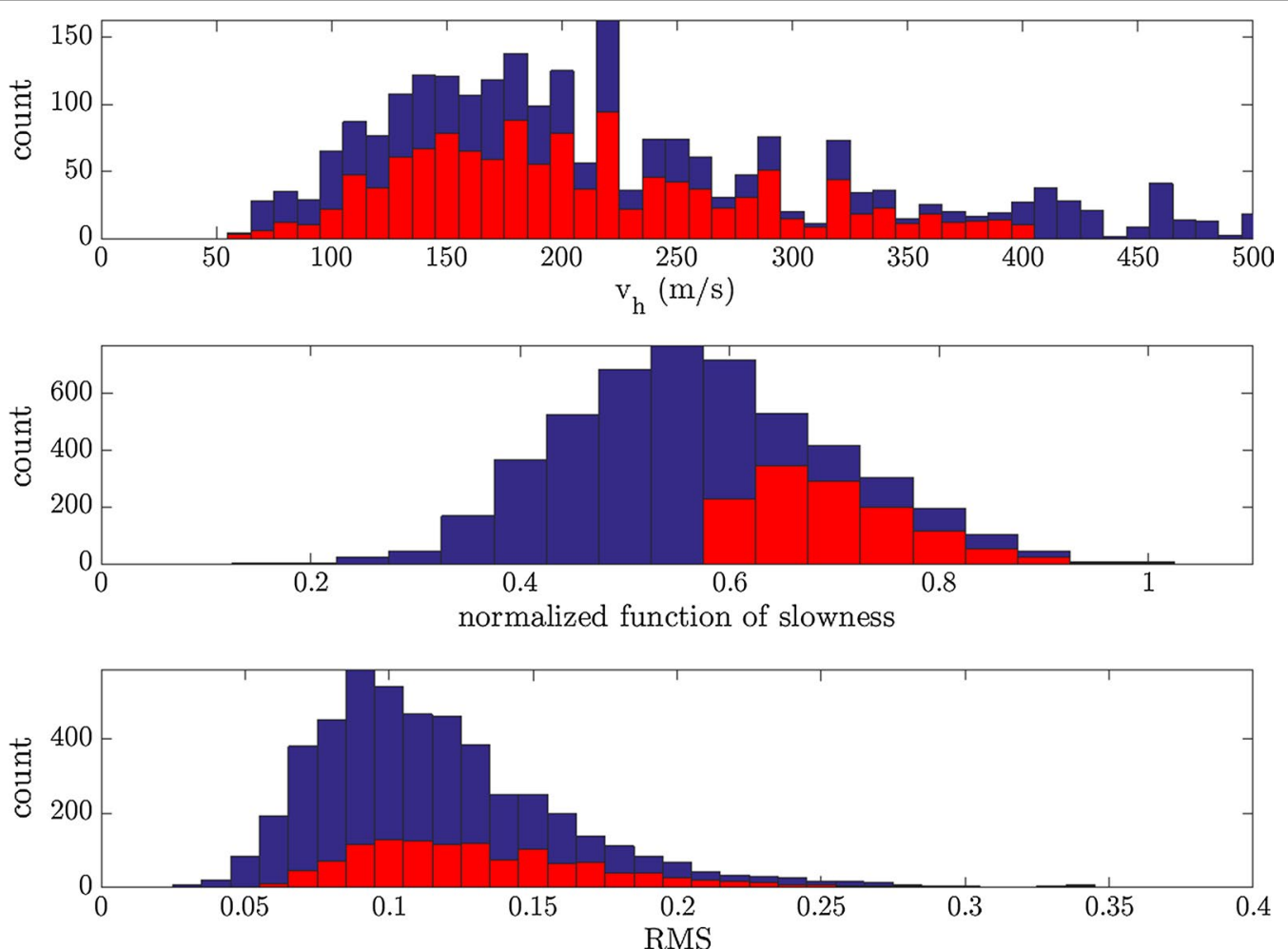

Fig. 5 (Top) distribution of observed GW horizontal velocities. (Middle) the distribution of normalized function $C\left(s_{x^{\prime}} s_{y}\right)$ maxima in the analyzed intervals. (Bottom) the distribution of RMS values in the analyzed intervals. Blue color represents all observed events. Red are events selected for statistical analysis based on the selection criteria

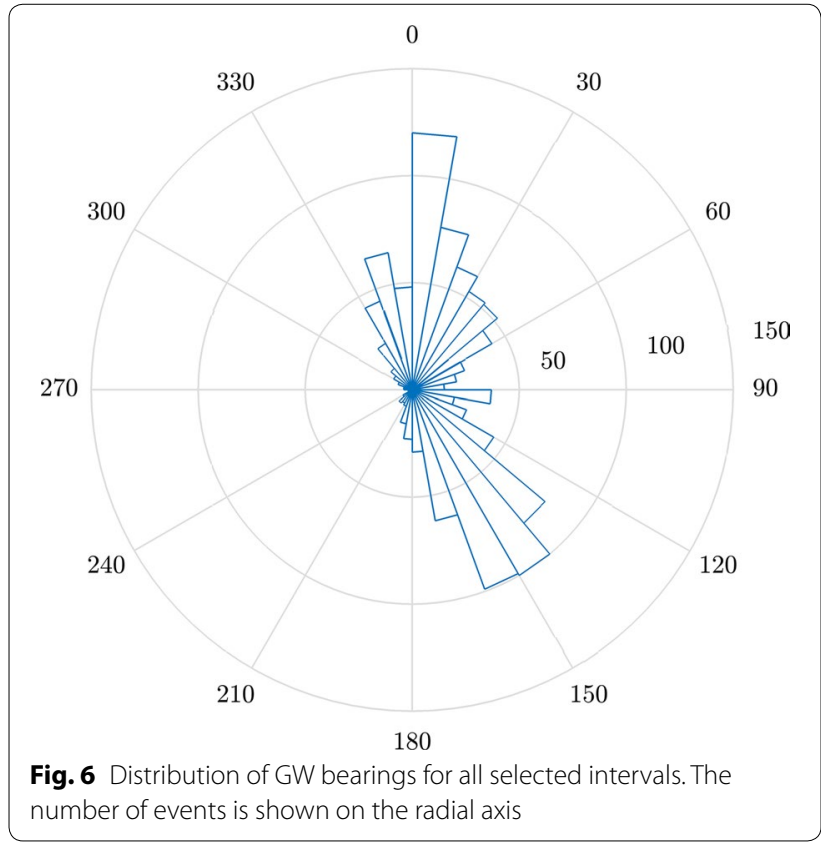

mostly in nighttime, and the meridional component of their propagation direction was also equatorward. This corresponds to our observations in the winter season. However, the zonal component was mostly westward in their observations, whereas it was mainly eastward in our observations and the MSTIDs persisted to evening hours. This difference might be caused by a different position of the MSTID source locations with respect to Taiwan and Central China and differences in local neutral winds. Their observations were also in a different time period. One case of nighttime MSTID over Taiwan that propagated to the southwest on May 26, 2006, was also reported by Lee et al. (2008) based on GPS-TEC observations.

Frissell et al. (2016) studied sources and characteristics of MSTIDs in the North American sector using HF radar. They focused on MSTIDs observed during the winter daytime. They found that a majority of MSTIDs propagated equatorward in this period, which is similar to our observations. Oinats et al. (2015) studied MSTIDs using the Super Dual Auroral Radar Network in Hokkaido, i.e., a location that 


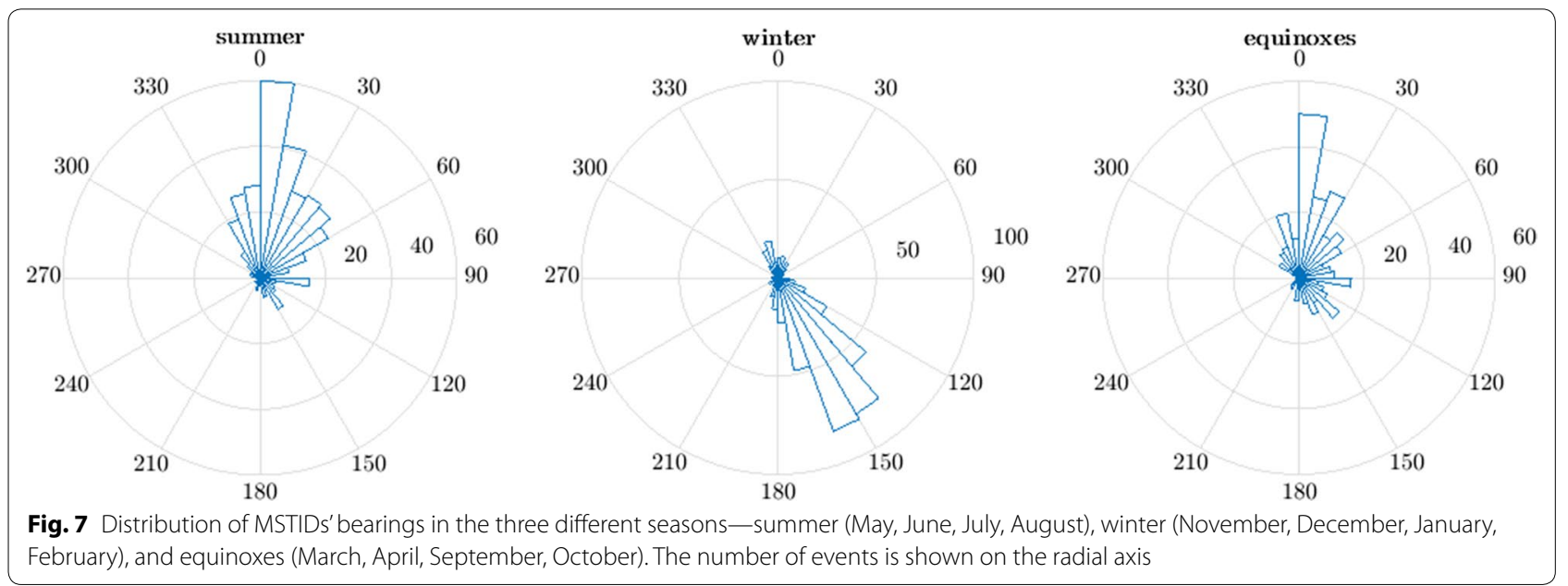
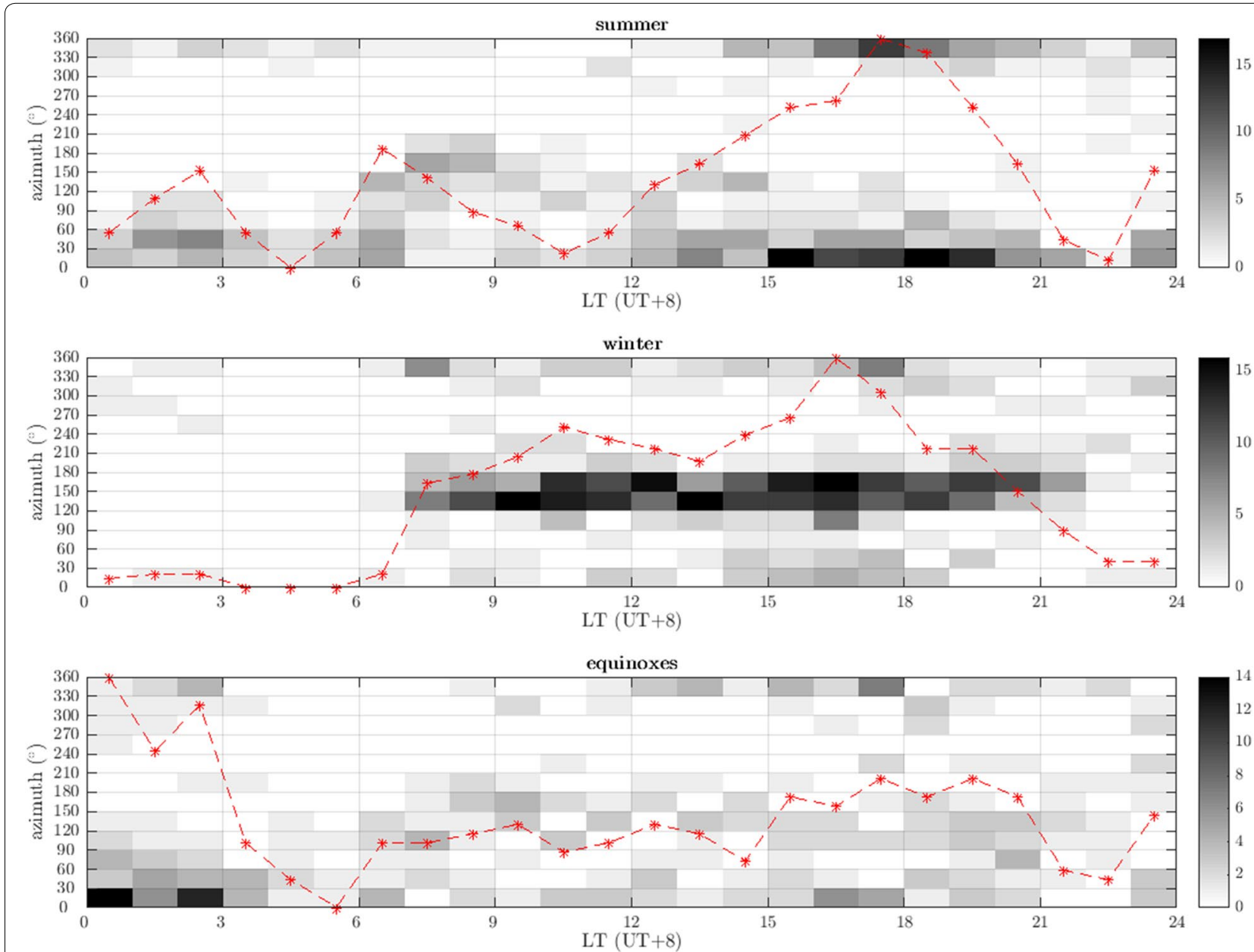

Fig. 8 Bivariate daytime azimuth histogram of MSTIDs occurrence. The observation time for each event corresponds to the middle of the analyzed 90-min interval. The red line depicts the sum of events in each hour over all directions normalized to the range $[0,360]$ 
is more poleward than Taiwan. Northeastward propagating MSTIDs were typical for summer and equinox. In Taiwan, we also observe northeastward propagating MSTIDs in summer; however, they are more often observed in the evening hours. Southeast azimuths prevailed in winter daytime. We also observe southeast azimuths in Taiwan in this period, as shown in Fig. 8. Oinats et al. (2015) also observed southwest azimuths in the summer, equinox nighttime, and equinox evening and northwest azimuths in summer daytime and evening; similar observations are not found in our observations from Taiwan.

Otsuka et al. (2013) studied MSTIDs over Europe using GPS in 2008. They found that MSTIDs can be categorized into two groups: daytime and nighttime. Daytime winter MSTIDs were observed to propagate southward, which is similar to our observations.

A histogram of differences between MSTIDs propagation directions and neutral wind horizontal directions calculated at the reflection heights and observation times using the HWM14 model (Drob et al. 2015) is shown in Fig. 9. It is calculated as a difference between the azimuth of observed MSTID and azimuth of modeled horizontal wind. For values less than zero, $360^{\circ}$ is added. The reflection heights are estimated using the IRI 2012 model (Bilitza et al. 2014). The MSTIDs mostly propagate against the modeled horizontal wind. This confirms that the observed MSTIDs were primarily due to GWs because the atmospheric winds pose a directional filter. This filter favors GWs propagating against the winds to reach ionospheric heights (Sun et al. 2007).

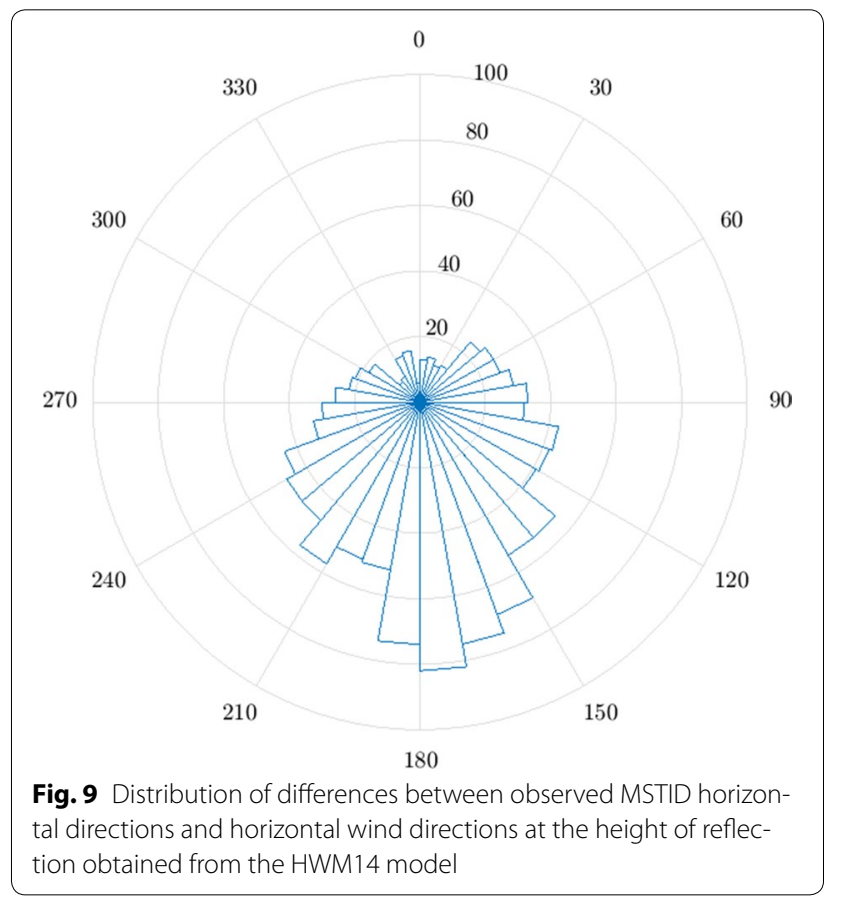

\section{Conclusions}

We analyzed horizontal velocities of MSTIDs using HF Doppler soundings over Taiwan from January 2014 to January 2016. Meridional components of the observed horizontal velocities are in most cases larger than zonal components. In summer, poleward propagation prevails, whereas in winter, disturbances propagate mostly southward. Zonal components of the analyzed events are mostly eastward. Seasonal behaviors of the observed propagation directions are similar to two other HF Doppler sounding locations at middle latitudes in Czechia Republic and South Africa and to low-latitude station in Tucumán, Argentina. Horizontal velocity of the observed events is in the $50-400-\mathrm{ms}^{-1}$ range. The upper limit is determined from the spacing between reflection points, increasing the uncertainty in velocity for events with velocities higher than this limit. The mean horizontal velocity is $210 \mathrm{~ms}^{-1}$. The range between the 20th and 80th percentile of horizontal velocities is $144-281 \mathrm{~ms}^{-1}$.

\section{Abbreviations}

GWs: Gravity waves; HF: High frequency; MSTIDs: Mesoscale traveling ionospheric disturbances.

\section{Author details \\ ${ }^{1}$ Institute of Atmospheric Physics, Czech Academy of Sciences, Bocni II 1401, Prague, Czech Republic. ${ }^{2}$ Faculty of Mathematics and Physics, Charles Univer- sity in Prague, Prague, Czech Republic. ${ }^{3}$ Institute of Space Science, National Central University, Chung-Li 320, Taiwan.}

\section{Authors' contributions}

JF wrote the manuscript and performed most of the data analysis. JC helped with writing of the manuscript and created the software for data processing. $J-Y L$ provided most of the data from Taiwan. All authors read and approved the final manuscript.

\section{Acknowledgements \\ The Doppler data are available at http://datacenter.ufa.cas.cz/ under the link to the Spectrogram archive. The IRI-2012, http://omniweb.gsfc.nasa.gov/vitmo/ irizo12 vitmo.html, is acknowledged for providing the electron density profile from which the reflection heights were obtained. The support under Grant 15-07281 J by the Czech National Foundation is acknowledged.}

\section{Competing interests}

The authors declare that they have no competing interests.

\section{Publisher's Note}

Springer Nature remains neutral with regard to jurisdictional claims in published maps and institutional affiliations.

Received: 20 February 2017 Accepted: 11 September 2017

Published online: 20 September 2017

\section{References}

Altadill D, Solé JG, Apostolov EM (2001) Vertical structure of a gravity wave like oscillation in the ionosphere generated by the solar eclipse of August 11, 1999. J Geophys Res 106(A10):21419. doi:10.1029/2001 JA900069

Artru J et al (2005) lonospheric detection of gravity waves induced by tsunamis. Geophys J Int 160(3):840-848. doi:10.1111/j.1365-246X.2005.02552.x 
Bertin F et al (1978) The meteorological jet stream as a source of medium scale gravity waves in the thermosphere: an experimental study. J Atmos Terr Phys 40(10-11):1161-1183. doi:10.1016/0021-9169(78)90067-3

Bilitza D et al (2014) The international reference ionosphere 2012 a model of inter-national collaboration. J Space Weather Space Clim 4:A07. doi:10.1051/swsc/2014004

Boška J et al (2003) Diurnal variation of gravity wave activity at midlatitudes in the ionospheric F region. Stud Geophys Geod 47(3):579-586. doi:10.102 3/A:1024763618505

Chou MY et al (2017) Concentric traveling ionosphere disturbances triggered by Super Typhoon Meranti (2016). Geophys Res Lett 44(3):1219-1226. doi :10.1002/2016GL072205

Chum J et al (2010) Horizontal velocities and propagation directions of gravity waves in the ionosphere over the Czech Republic. J Geophys Res Space Phys 115:1-13. doi:10.1029/2010JA015821

Chum J et al (2012) Statistical investigation of horizontal propagation of gravity waves in the ionosphere over Europe and South Africa. J Geophys Res Space Phys 117(3):1-13. doi:10.1029/2011JA01716

Chum J et al (2014) Propagation of gravity waves and spread F in the lowlatitude ionosphere over Tucumán, Argentina, by continuous Doppler sounding: first results. J Geophys Res Space Phys 119(8):6954-6965. doi:1 0.1002/2014JA020184

Chum J et al (2016) lonospheric signatures of the April 25, 2015 Nepal earthquake and the relative role of compression and advection for Doppler sounding of infrasound in the ionosphere. Earth Planets Space 68(1):24 doi:10.1186/s40623-016-0401-9

Crowley G, Rodrigues FS (2012) Characteristics of traveling ionospheric disturbances observed by the TIDDBIT sounder. Radio Sci 47:RSOL22. doi:10.10 29/2011RS004959

Davies K, Baker DM (1966) On frequency variations of ionospherically propagated HF radio signals. Radio Sci 1(5):545-556. doi:10.1002 rds196615545

Ding F et al (2011) Climatology of medium-scale traveling ionospheric disturbances observed by a GPS network in central China. J Geophys Res Space Phys 116(9):1-11. doi:10.1029/2011JA016545

Drob DP et al (2015) An update to the Horizontal Wind Model (HWM): the quiet time thermosphere. Earth Space Sci. doi:10.1002/2014EA000089

Frissell NA et al (2014) Climatology of medium-scale traveling ionospheric disturbances observed by the midlatitude Blackstone SuperDARN radar. $J$ Geophys Res Space Phys 119(9):7679-7697. doi:10.1002/2014JA019870

Frissell NA et al (2016) Sources and characteristics of medium scale traveling iono-spheric disturbances observed by high frequency radars in the North American sector. J Geophys Res Space Phys 121(4):3722-3739. doi: 10.1002/2015JA022168

Fritts DC, Lund TS, Hultqvist B (2011) Gravity wave influences in the thermosphere and ionosphere: observations and recent modeling. In: Abdu MA, Pancheva D (eds) Aeron. Earth's atmos ionos (Chap. 8). Springer, Dordrecht, pp 109-130. doi:10.1007/978-94-007-0326-1

Fukushima D et al (2012) Observation of equatorial nighttime medium-scale traveling ionospheric disturbances in 630-nm airglow images over 7 years. J Geophys Res Space Phys 117(10):1-13. doi:10.1029/2012JA017758

Georges TM (1968) HF Doppler studies of TIDs. J Atmos Terr Phys 30(5):735746. doi:10.1016/50021-9169(68)80029-7

Grocott A et al (2013) Characteristics of medium-scale traveling ionospheric disturbances observed near the Antarctic Peninsula by HF radar. J Geophys Res Space Phys 118(9):5830-5841. doi:10.1002/jgra.50515

He L-S et al (2004) Studies of medium scale travelling ionospheric disturbances using TIGER SuperDARN radar sea echo observations. Ann Geophys 22(12):4077-4088. doi:10.5194/angeo-22-4077-2004

Hernández-Pajares M et al (2012) Propagation of medium scale traveling ionospheric disturbances at different latitudes and solar cycle conditions. Radio Sci 47:4. doi:10.1029/2011RS004951

Hines CO (1960) Internal atmospheric gravity waves at ionospheric heights. Can J Phys 38(11):1441-1481. doi:10.1139/p60-150

Hocke K, Schlegel K (1996) A review of atmospheric gravity waves and travelling ionospheric disturbances: 1982-1995. Ann Geophys 14(9):917-940. doi:10.1007/s00585-996-0917-6

Huang Y-N, Cheng K, Chen S-W (1985) On the detection of acoustic-gravity waves generated by typhoon by use of real time HF Doppler frequency shift sounding system. Radio Sci 20(4):897-906. doi:10.1029/ RS020i004p00897
Hunsucker RD (1982) Atmospheric gravity waves generated in the highlatitude iono-sphere: a review. Rev Geophys 20(2):293. doi:10.1029/ RG020i002p00293

Ichihara A et al (2013) Northward-propagating nighttime medium-scale traveling iono-spheric disturbances observed with SuperDARN Hokkaido HF radar and GEONET. Adv Polar Sci 24(1):42-49. doi:10.3724/ SP.J.1085.2013.00042

Ishida T et al (2008) SuperDARN observations of daytime MSTIDs in the auroral and mid-latitudes: possibility of long-distance propagation. Geophys Res Lett 35(13):L13102. doi:10.1029/2008GL034623

Johnson JB et al (2011) Imaging thunder. Geophys Res Lett. doi:10.1029/201 1GL049162

Kelley MC (2011) On the origin of mesoscale TIDs at midlatitudes. Ann Geophys 29(2):361-366. doi:10.5194/angeo-29-361-2011

Klausner V et al (2009) Observations of GW/TID oscillations in the F2 layer at low latitude during high and low solar activity, geomagnetic quiet and disturbed periods. J Geophys Res Space Phys 114(2):1-11. doi:10.1029/2 008JA013448

Kotake N, Otsuka Y (2007) Statistical study of medium-scale traveling ionospheric disturbances observed with the GPS networks in Southern California. Earth Planets Space 59(2):95-102. doi:10.1007/978-94-007-0326-1_21

Laštovička J (2009) Lower ionosphere response to external forcing: a brief review. Adv Space Res 43:1-14. doi:10.1016/j.asr.2008.10.001

Lay EH et al (2015) lonospheric acoustic and gravity waves associated with mid-latitude thunderstorms. J Geophys Res Space Phys 120:6010-6020. doi:10.1002/2015JA021334

Lee CC et al (2008) Nighttime medium-scale traveling ionospheric disturbances detected by network GPS receivers in Taiwan. J Geophys Res Space Phys 113(12):1-6. doi:10.1029/2008JA013250

Liu H et al (2014) Gravity waves simulated by high-resolution Whole Atmosphere Community Climate Model. J Geophys Res Atmos 41:9106-9112. doi:10.1002/2014GL062468.For

MacDougall J et al (2011) Spaced transmitter measurements of medium scale traveling ionospheric disturbances near the equator. Geophys Res Lett 38(16):1-6. doi:10.1029/2011GL048598

Medvedev AV et al (2013) Studying of the spatial-temporal structure of wavelike ionospheric disturbances on the base of Irkutsk incoherent scatter radar and Digisonde data. J Atmos Solar Terr Phys 105-106:350-357. doi:10.1016/j.jastp.2013.09.001

Oinats AV, Kurkin VI, Nishitani N (2015) Statistical study of medium-scale traveling ionospheric disturbances using SuperDARN Hokkaido ground backscatter data for 2011. Earth Planets Space 67(1):22. doi:10.1186/ s40623-015-0192-4

Oinats AV et al (2016) Statistical characteristics of medium-scale traveling ionospheric disturbances revealed from the Hokkaido East and Ekaterinburg HF radar data. Earth Planets Space 68(1):8. doi:10.1186/ s40623-016-0390-8

Otsuka Y et al (2011) Statistical study of medium-scale traveling ionospheric disturbances observed with a GPS receiver network in Japan. In: Aeron Earth's atmos ionos. Springer, Dordrecht, pp 291-299. doi:10.1007/978-94-007-0326-1_21

Otsuka Y et al (2013) GPS observations of medium-scale traveling ionospheric disturbances over Europe. Ann Geophys 31 (2):163-172. doi:10.5194/ angeo-31-163-2013

Šauli P et al (2006) Detection of the wave-like structures in the F-region electron density: two station measurements. Stud Geophys Geod 50(1):131146. doi:10.1007/s11200-006-0007-y

Shiokawa K et al (2003) Statistical study of nighttime medium-scale traveling iono-spheric disturbances using midlatitude airglow images. J Geophys Res Space Phys 108(A1):1-7. doi:10.1029/2002JA009491

Shiokawa K, Otsuka Y, Ogawa T (2009) Propagation characteristics of nighttime mesospheric and thermospheric waves observed by optical mesosphere thermosphere imagers at middle and low latitudes. Earth Planets Space 61:479-491. doi:10.1186/BF03353165

Sun L et al (2007) Gravity wave propagation in the realistic atmosphere based on a three-dimensional transfer function model. Ann Geophys 25(9):1979-1986. doi:10.5194/angeo-25-1979-2007

Sutcliffe PR, Poole AWV (1989) lonospheric Doppler and electron velocities in the presence of ULF waves. J Geophys Res 94:13505. doi:10.1029/ JA094iA10p13505 
Waldock J, Jones T (1987) Source regions of medium scale travelling ionospheric disturbances observed at mid-latitudes. J Atmos Terr Phys 49(2):105-114. doi:10.1016/0021-9169(87)90044-4

Wan W et al (1998) Traveling ionospheric disturbances associated with the tropospheric vortexes around Qinghai-Tibet Plateau. Geophys Res Lett 25(20):3775. doi:10.1029/1998GL900030
Wautelet G, Warnant R (2015) Origin of high-frequency TEC disturbances observed by GPS over the European mid-latitude region. J Atmos Solar Terres Phys 133:67-78. doi:10.1016/j.jastp.2015.08.003

Yu Y, Hickey MP (2007) Numerical modeling of a gravity wave packet ducted by the thermal structure of the atmosphere. J Geophys Res Space Phys 112(6):1-12. doi:10.1029/2006JA012092 